\title{
Surgical Treatment of Giant Cavernous Hepatic Haemangiomas
}

\author{
Ibrahim Abdelkader Salama $^{{ }^{*}}$, Mohammed Hussein Abdullah ${ }^{2}$, Mohammed Houseni $^{3}$ \\ ${ }^{1}$ Department of Hepatobilary Surgery, National Liver Institute, Menophyia University, Shiben Elkom, Egypt \\ ${ }^{2}$ Department of Anesthesia, National Liver Institute, Menophyia University, Shiben Elkom, Egypt \\ ${ }^{3}$ Department of Radiology, National Liver Institute, Menophyia University, Shiben Elkom, Egypt \\ Email: *ibrahim_salama@hotmail.com
}

Received November 17, 2011; revised January 11, 2012; accepted February 23, 2012

\begin{abstract}
Background: Haemangiomas are the most common benign liver tumors. Treatment is indicated for symptomatic tumors, rapid increase in size, rupture or doubt in diagnosis. Objective: Evaluation the efficacy of surgical treatment of giant cavernous hepatic haemangioma in tertiary hepatobiliary center. Patients and Methods: Retrospective study of 34 patients with giant hepatic haemangioma operated upon. The diagnosis was proved preoperatively in 27 patients and confirmed by histopathology postoperatively in all patients. The indication of surgery was abdominal pain with large sizes tumors, rapid growth, and spontaneous rupture with haemoperitoneum. Surgical treatment either liver resection or living liver transplantation. Results: 23 cases (67.6\%) were females \& 11 cases (32.4\%) were males, median age 38.8 years. Haemangiomas located in right lobe in 19 patients (55.9\%), left lobe in 12 patients (35.4\%) in both lobe in 2 patients (5.8\%), scattered all over both lobe in one patient (2.9\%), solitary in 27 cases (79.4\%) and multiple in 7 patients (20.6\%). The diameter was $8-27 \mathrm{~cm}$, mean $18.6 \mathrm{~cm}$ in diameter. 33 patients had liver resection (29 elective resection and 4 emergent resection for rupture). One patient had haemangioma irresectable; the living liver transplant was performed. No mortality during 18 months follow up and complication occurred in 8 patients out of 34 patients. Conclusion: Hepatic resection is an effective treatment option for giant cavernous hepatic haemangioma, in selected patient living related liver transplantation may be the only therapeutic option.
\end{abstract}

Keywords: Liver Neoplasms; Haemangioma; Hepatectomy

\section{Introduction}

Haemangiomas are the most common benign tumors of the liver [1]. The lesions, which may be single or multiple, are thought to be vascular malformations that enlarge by means of ectasia rather than neoplastic growth [2]. Those with a diameter greater than $4 \mathrm{~cm}$ have been refereed to as giant haemangiomas which may present as a symptomatic abdominal mass [3]. Although most haemangiomas are asymptomatic and may be managed safely with observation alone, larger lesions may produce a variety of symptoms and signs, including pain (abdominal, back or shoulder), fullness, early satiety, nausa, vomiting, and fever or more rarely haemangioma may rupture or be associated with Kasabach-Merritt Syndrome with thrombocytopenia secondary to platelet trapping within the haemangioma, or abscess formation [4].

Strategies for the management of liver haemangioma have ranged from selective observation to a variety of radiological and surgical interventions [5]. While there is general agreement that small asymptomatic lesions should

\footnotetext{
${ }^{*}$ Corresponding author.
}

be managed conservatively, the surgical resection provides the only consistently effective method of treatment and is indicated for symptomatic lesions in patients with an acceptable surgical risk and lesions for which a diagnosis is equivocal despite appropriate preoperative evaluation [2]. The unknown natural history and the possibility of complications of larger or giant haemangiomas may pose therapeutic dilemma [4].

The aim of this present study is to evaluate the efficacy of surgical treatment of giant cavernous haemangioma of the liver in our tertiary hepatobiliary center.

\section{Patients and Methods}

This is a retrospective study on 34 patients with giant haemangiomas of the liver who underwent surgical operation at National liver Institute (Tertiary hepatobiliary center), Menophyia University between March 2005 to March 2010. The following investigations were performed for all patients: laboratory tests (complete blood pictures, liver function tests, coagulation profiles, CA 19-9 and alpha-fetoprotein) imaging studies includes abdominal 
ultrasonography, Triphasic Spiral Computed Tomography (CT), (Figures 1(A) and (B)) and sometimes Magnetic Resonance Imaging (MRI) (in 8 patients only). The current MRI protocol for haemangiomaa includes T2-weighted images with short and long echo times, Multiphasic dynamic contrast-enhanced T1-weighted images and delayed fat-saturated dynamic contrast-enhanced T1-weighted images (Figures 2(A) and (B)).

The diagnosis was proved preoperatively in 27 patients by combination of typical findings in imaging studies and clinical symptoms and signs. Post-operatively, the diagnosis was confirmed by gross and histopathological examination in all patients who underwent surgical resection.

The indication for surgery was abdominal pain with large sized tumors, rapid growth or increase in size, and spontaneous rupture with haemoperitoneum and shock (4 cases).

A variety of surgical procedures were employed, designed to minimize the unnecessary loss of normal liver tissue. Surgical treatment consisted of either liver resection or living related liver transplantation.
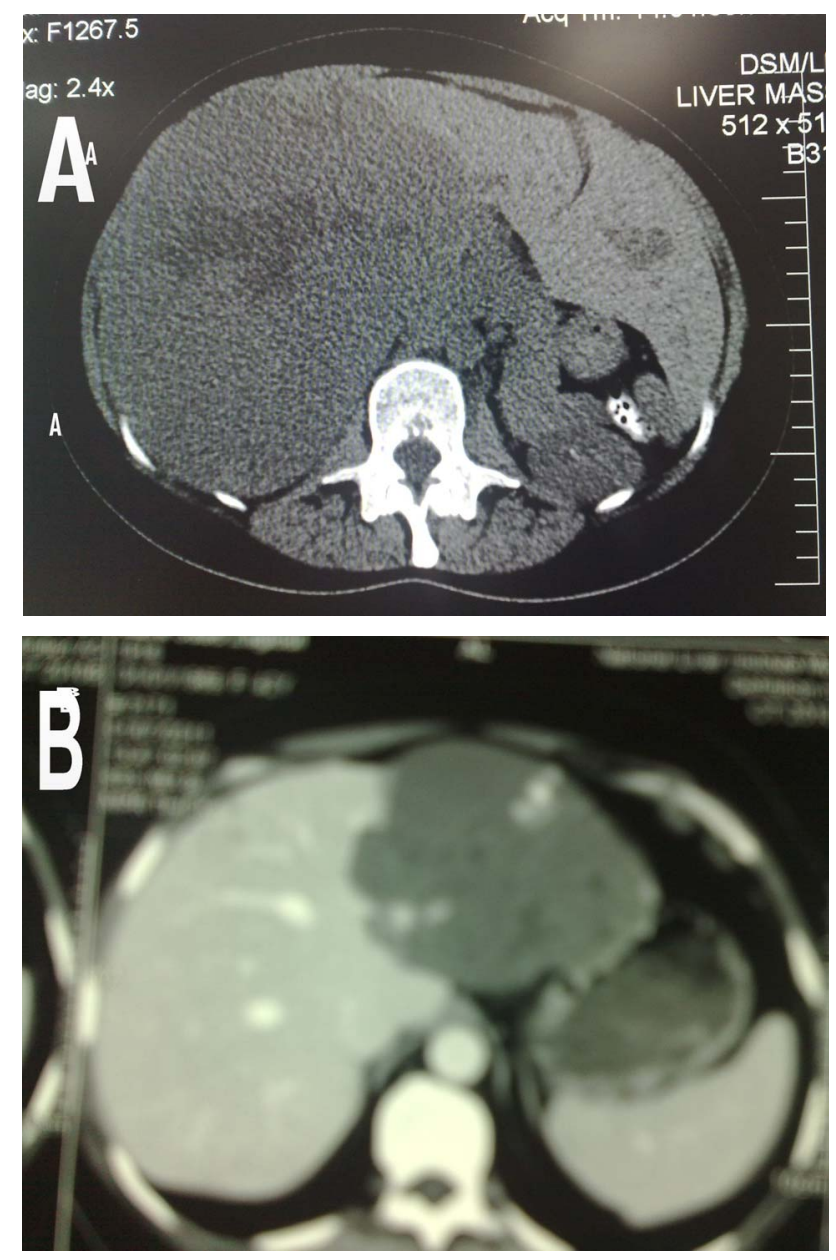

Figure 1. Non-enhanced CT image of giant hepatic haemangioma. (A) Right lobe; (B) Left lobe.
The formal liver resection started in non-urgent cases with abdomen explores through a Chevron incision. The liver was mobilized by division of ligamentous attachments; care was taken to preserve the left triangular ligament in case of right hepatectomy. The haemangioma was identified and a decision as to the type of resection was made, based on the location of the lesion(s) and size in relation to the volume of normal parenchyma. To reduce bleeding in large resection, a Pringles' maneuver was performed by placing a non crushing vascular clamp across the porta hepatis. Inflow was occluded for no more than 20 minutes, at which point the clamp was released and the liver perfused for 5 minutes, if necessary, the clamp was then reapplied. After identification and subsequent ligation and division of the portal structures at the hilum of the liver (hepatic artery, portal vein, and hepatic duct) of segement, that had to be resected. Therafter, identification and ligation of the hepatic vein(s) was performed, with identification of the transaction plane guided by the discoloration of the devascularized parts of the liver. Trans-section of the liver parenchyma was undertaken either by a Cavitaron Ultrasound Surgical Aspirator (CUSA) (Valley Lab,
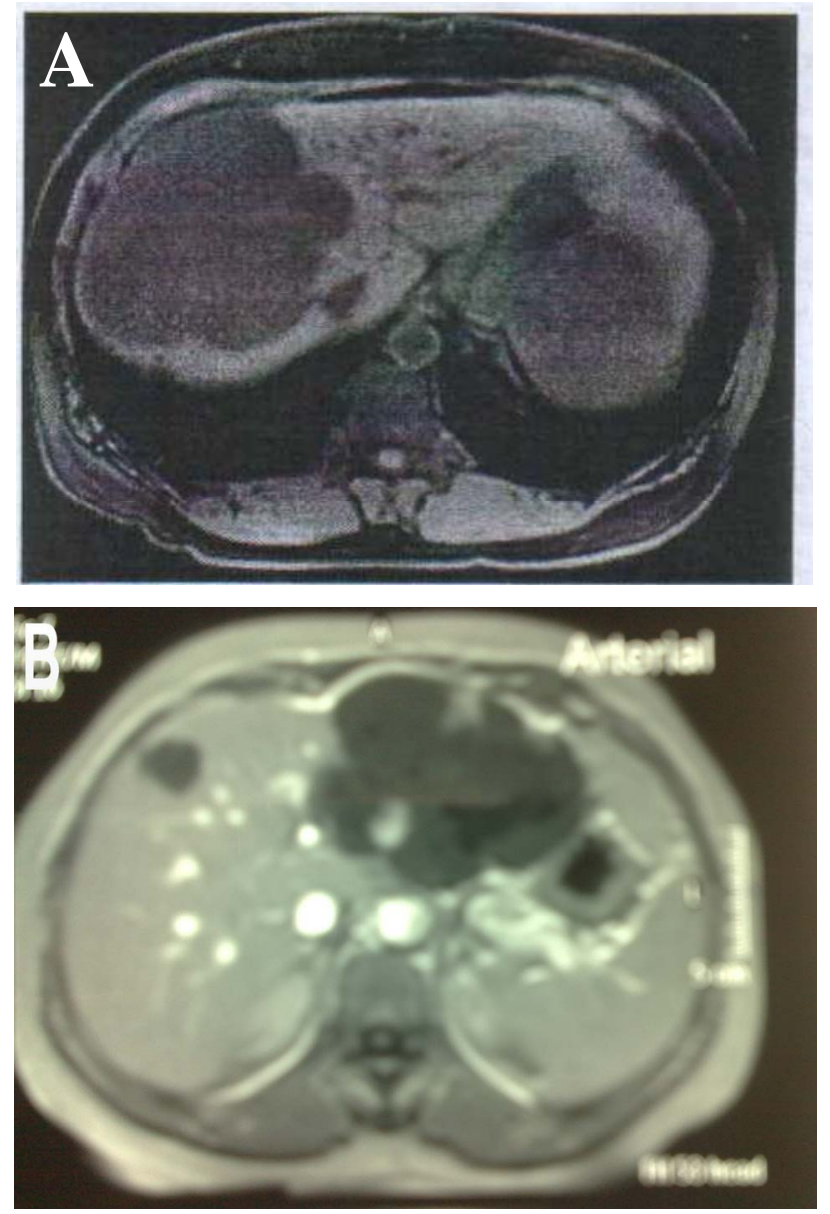

Figure 2. MRI image of giant hepatic haemangioma. (A) Right lobe; (B) Left lobe. 
Boulder, Colo, USA) with bipolar diathermy or Harmonic Scalpel (Harmonic Scalpel, Ethicon Endo-Surgery, Cincinnati, OH, USA) (Figures 3(A) and (B)).

After completion of the operative resection the haemostasis was done to raw surface of the liver (by organ beam or diathermy cautry) and small bile ducts were closed and abdomen closed with drain in most of cases.

Four patients underwent emergency operations because shock and haemoperitoneum due to rupture of giant haemangioma of the liver. One patient from those 4 emergency patients referred from other hospital after exploration (this patient experienced sudden onset of abdominal pain and tenderness in the right hypochondrium, there was no history of trauma, or significant disease in the past. The patient underwent laparotomy owing to a misdiagnosis of rupture liver abscess; on exploration haemoperitoneum with active bleeding from the liver haemangioma was found. Due to lack of experience, perihepatic packing was done with sponges, and patient was referred to our tertiary care institute. After the patient stabilized haemodynamically, the patient underwent spiral CT revealed a peripherally enhancing lesion in right lobe of the liver, located in
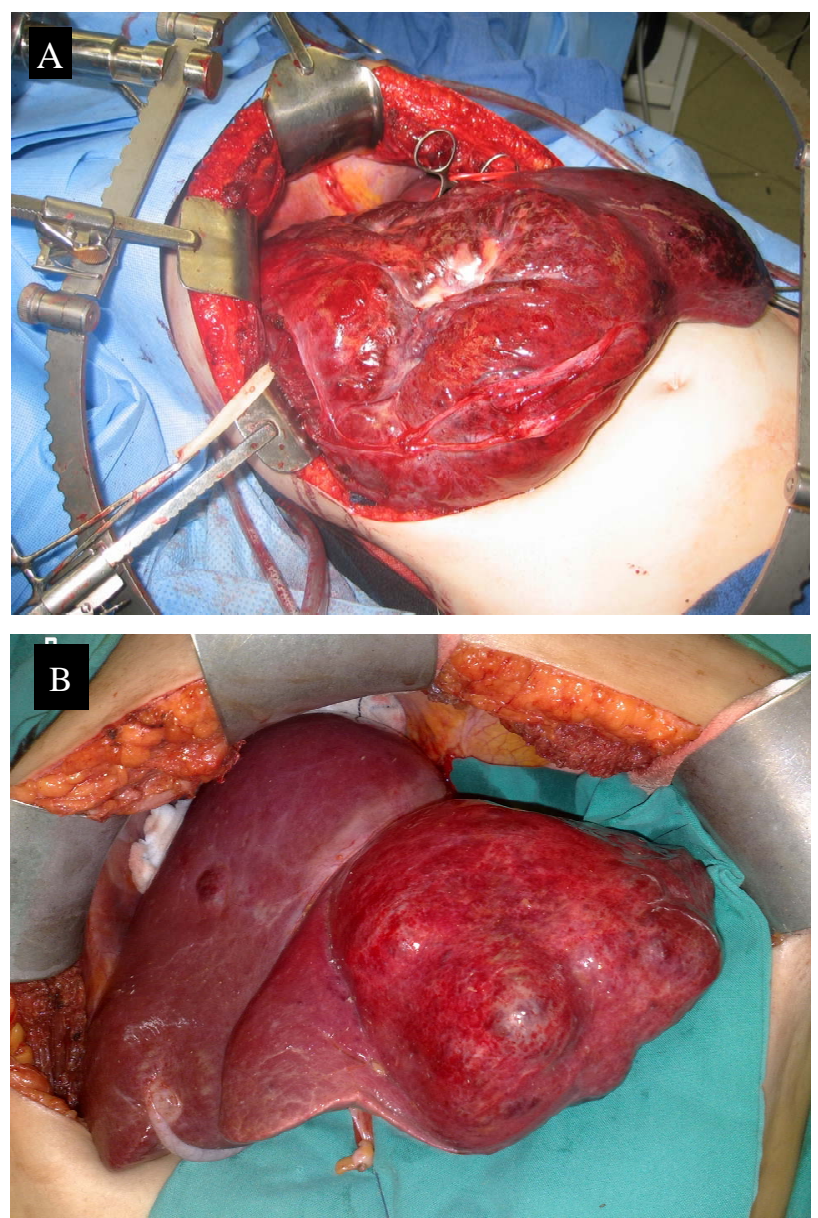

Figure 3. Intraoperative giant hepatic haemangioma. (A) Right lobe; (B) Left lobe. segments VI, VII \& VIII the patient underwent formal right hepatectomy (Figures 4(A) and (B)).

In one case (child girl) underwent total hepatectomy with left lateral segment living related liver transplantation from her mother (Figures 5(A) and (B)).

\subsection{Follow-Up}

Postoperative examination in the patients consisted of physical examination, liver function tests and ultrasonography of the remnant liver, the patient follow up at 6 months interval for 18 months after liver resection, patient with living liver transplantation was seen each Month as protocol of liver transplantation unit in the institute.

\subsection{Statistical Analysis}

Statistical analysis were performed using the Student-t test for continuous variables, continuous variables were expressed as mean \pm SD. A P value of less than 0.05 was considered significant.
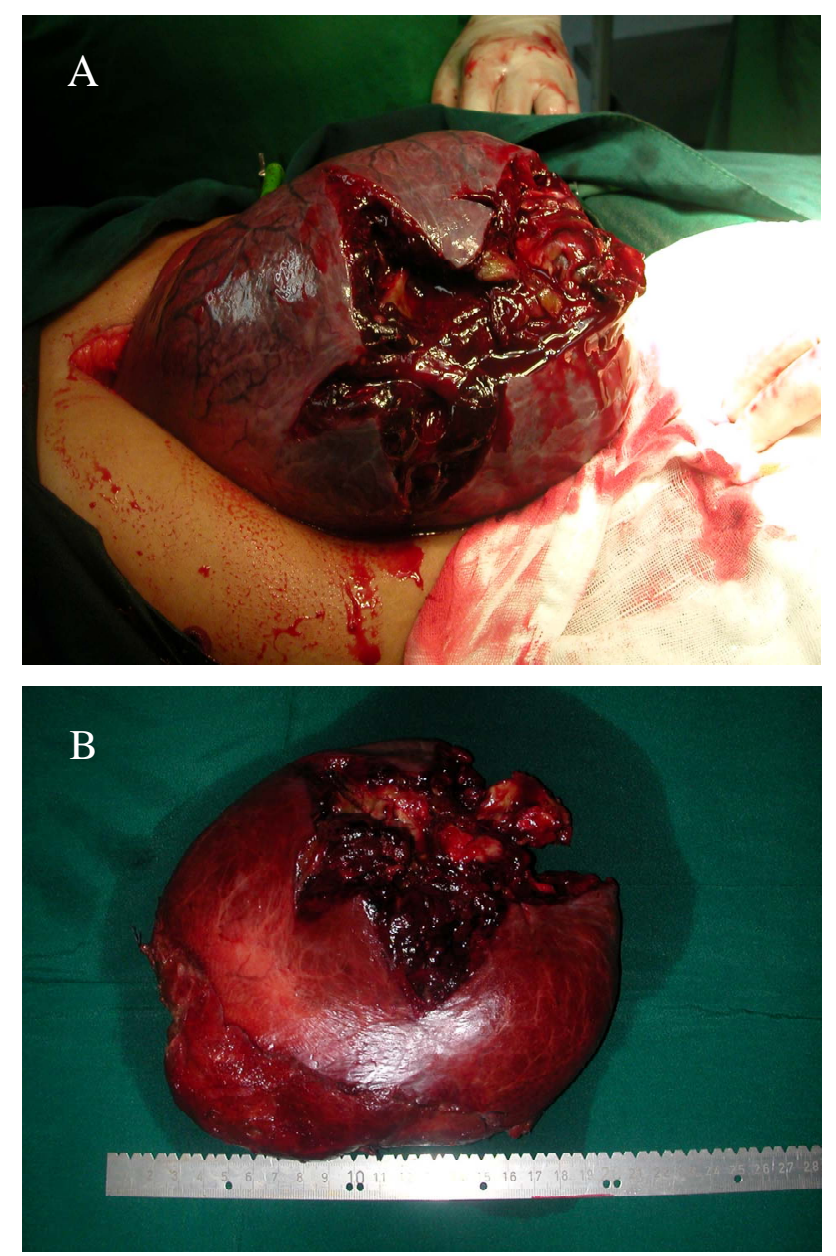

Figure 4. A case of rupture cavernous right lobe hepatic haemangioma. (A) Intraoperative image; (B) Excised haemangioma. 

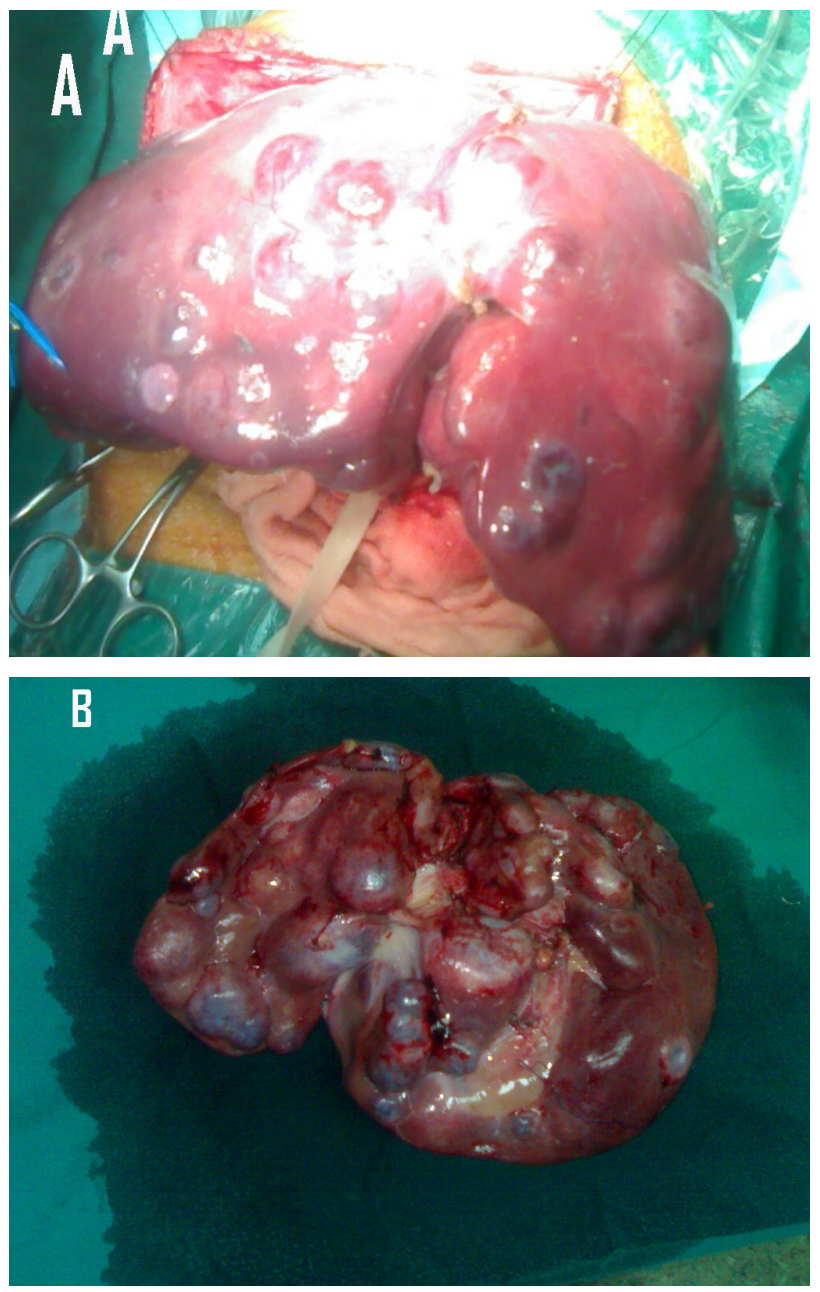

Figure 5. A case of multiple cavernous haemangioma in $\mathbf{1 . 5}$ year-old girl. (A) Intraoperative image; (B) Excised liver.

\section{Results}

\subsection{Patient Demographics}

During the study period. 34 patients underwent surgical removal of giant cavernous hepatic haemangiomas, 23 cases (67.6\%) were females and 11 cases (32.4\%) were males with age range from 1.5 - 55 years and median 38.8 years.

\subsection{Clinical Findings}

The majority of patients were symptomatic (27 cases) complaining of upper abdominal pain, pressure symptoms due to increase the size of the tumors such as fullness and dyspepsia. The remaining 7 patients were asymptomatic, 4 out of them were presented with acute abdomen \& shock as the first presentation.

Physical examination revealed hepatomagly in 22 cases, hepatomagly and palpable liver mass in the remaining cases. Laboratory tests were normal in all but 5 patients (anemia was found in 3 patients and abnormal liver func- tion test in 2 patients).

None of patients had consumptive coagulopathy based on the routine haematological parameters, detailed coagulation parameters were not assessed in the absence of specific symptoms.

Eleven patients had associated co-morbid conditions, including hypertension in 4 patients, diabetes in 3 patients, coronary artery diseases in 2 patients, gall stone and renal calculus in one each.

\subsection{Characteristics of Haemangiomas}

Giant Cavernous hepatic haemangiomas were located in the right lobe in 19 patients (55.9\%), in the left lobe in 12 patients (35.4\%), in both lobe in 2 patients (5.8\%) and scattered all over both right and left lobe of the liver in one patient (2.9\%). Haemangiomas were identified as solitary lesion in 27 cases (79.4\%) and multiple in 7 patients (20.6\%). The size of detected haemangioma was ranging from 8 to $27 \mathrm{~cm}$ in its largest diameter with a mean diameter of $18.6 \mathrm{~cm}$.

\subsection{Operative Procedures}

30 patients underwent elective surgery while in 4 patients an emergency partial hepatectomy had to be performed because of a ruptured haemangioma (3 in the right lobe (right hepatectomy was done) and one in the left lobe (left lateral segmentectomy was performed).

In one patient (1.5 years old girl) had multiple haemangiomas in both lobes of the liver make the patient unable to move or lie down without pain due to large mass in upper abdomen, these multiple irresectable haemangiomas in both lobes with this symptoms were indicated for living liver transplant (mother donate to her daughter).

The types of liver resection performed among patients in the present study group are shown in Table $\mathbf{1}$.

In multiple haemangiomas only the lesions responsible for the symptoms were resected, in all patients this appeared to be the largest lesion. In one patient with giant haemangioma had another haemangioma in ipsilateral lobe was resected with giant haemangioma, in one patient only the largest lesions were resected and the additional smaller haemangiomas were located in the contralateral lobe were

Table 1. Types of liver resection performed among patients in the study group.

\begin{tabular}{cc}
\hline Extent of resection & No. of patients \\
\hline Right formal hepatectomy & 14 \\
Left formal hepatectomy & 5 \\
Extended right hepatectomy & 5 \\
Left lateral segmentectomy & 7 \\
Enucleation & 2 \\
Total hepatectomy and left lateral segment LRLTx & 1 \\
\hline
\end{tabular}

LRLTx: Living related liver transplantation. 
left in-situ.

Pringle's inflow occlusion was performed during transaction of the liver in 12 patients. The median total inflow occlusion by the Pringles maneuver was 20 (ranging 10 to 37 ) minutes, median operative blood loss was one liter (ranging from $500 \mathrm{ml}$ to 2.5 liters), the mean postoperative stay was 7 days (ranging 4 to 16 days).

\subsection{Mortality and Morbidity}

There were no early or late deaths in this study.

Complications were occurred in 8 resected patients, minor biliary leakage (3 patients), pleural effusion (2 patients), wound infection (2 patients) and one patient had postoperative bleeding treated conservatively.

\subsection{Postoperative Follow-Up}

All patients followed up for 18 months, no recurrence of haemangioma was observed. In the patient with haemangioma left in-situ, no notable changes in the size of the remaining haemangioma in the liver remnant was found during the follow up period. The pediatric patient, who underwent living related liver transplantation, she recovered uneventfully, and was following up at outpatient clinic for liver transplantation at monthly interval without any significant complication.

\section{Discussion}

Cavernous haemangiomas occur a prevalence of $0.4 \%$ to $7.3 \%$ in autopsy series [1]. Cavernous haemangiomas are the most common benign neoplasm of the liver and are often incidentally detected during abdominal imaging done for unrelated clinical indications [6]. The lesions have female predominance with sex ratio is $1.5: 1$ consistent with the present study the female predominant (females were 23 cases (67.6\%), and male 11 cases (32.4\%) with ratio 1.8:1), these lesions are seen in all age groups but most frequently in middle age [7]. Diagnosis is confirmed by results of noninvasive radiological studies, dynamic contrast-enhanced magnetic resonance imaging is the best study, as it will identify smaller and/or multiple lesion [8].

The management of haemangioma is controversial and is intimately related to the size, symptoms and associated co morbidities of the patient who harbor these benign tumors [9].

Symptomatic haemangiomas are large and associated with a constellation of vague upper abdominal complaints including pain, masses, distention, early satiety and weight loss [9].

Symptomatic giant haemangiomas require some form of treatment as radiation, arterial embolization, surgical resection, or liver transplantation $[10,11]$.

The wide variability of symptoms in patients with haemangioma can be explained by known spontaneous regression, growth and rupture [12].

In the present study, giant haemangiomas were identified as solitary lesion in 27 patients (79.4\%) and multiple haemangiomas in the remaining 7 patients (20.6\%). The size of detected haemangioma was ranging between 8 to $27 \mathrm{~cm}$ with a mean diameter of $18.6 \mathrm{~cm}$. the majority of patients were symptomatic complaining of upper abdominal pain, also symptoms due to fullness and dyspepsia, these results compares favorably with Mergo and Ross [12].

Indications for operation have traditionally been the presence of symptoms, the development of symptoms and the need to establish a definite diagnosis when radiological and histological studies were inconclusive [4]. In the present study, the indications for surgical operations were due to symptomatic large-sized tumors, rapid increase in size and development of complication (spontaneous rupture).

Liver haemangiomas have also been resected because of a perceived risk of spontaneous or traumatic rupture and possibility of the Kasabach-Merritt syndrome, a consumption coagulapathy with low platelet counts and hypofibrinogenomia [4].

Intraoperitoneal bleeding from rupture, which can either occur spontaneously or due to trauma, is a rare lifethreating complication [5]; it occurs in about $4 \%$ of patients but is fatal in $60 \%-75 \%$ [13]. In a recent review of literature, the operative mortality rate of ruptured haemangioma was reported to be $36.4 \%$ [14].

In the current study, 4 patients out of 34 patients with giant haemangioma were presented with acute abdomen shock and haemoperitoneum due to ruptured giant hepatic haemangiomas, 3 patients in right lobe 21, 32, 27 $\mathrm{cm}$ in diameter respectively and one in the left lobe measuring $18 \mathrm{~cm}$ in diameter all managed by emergency liver resection successively.

Warmann and associates [15] do not consider the risk of rupture by itself an indication for resection. However, because of the high risk of a fatal outcome after rupture of such haemangiomas and in view of the low operative risks of partial liver resection Zeng and associates [16] believe that the operation is indicated even for asymptomatic giant haemangioma. This is particularly the case for patients with risk factors for rupture, such as superficial localization of the lesion in the liver haemangioma of large size, use of antithrombitic medication and the profession of the patient [17].

Specific feature of cavernous liver haemangioma may be apparent with a variety of imaging techniques such as ultrasonography, dynamic contrast-enhanced CT and MRI, with ultrasonography, a typical haemangioma is characterized as a sharply marginated, lobulated, perdomintelly hyperechoic lesion. However, utrasonography is an operator-dependent technique and the acquisition of satisfactory 
images in obese patients is technically difficult [12].

Multiphasic CT scanning should include arterial, portal venous phase and delayed imaging to ensure visualization of vascular structures. On non-enhanced CT scans, haemangimas appeared hypoattenuating relative to the adjacent liver. During the arterial-dominant phase, haemangiomas demonstrated a characteristic peripheral nodular and intense enhancement [18]. In patient with unclear diagnosis and in those with a known colorectal malignancy or cirrhosis, MRI was recommended because of its high specificity in the diagnosis of haemangioma [19].

The results of MRI in present study, although obtained only in 8 patients with haemangioma are in accordance with other previous report [20] the use of other invasive imaging methods, such as scientigraphy and technetium99 m labeled red blood cell scanning, has also been reported for the diagnosis of benign liver lesions especially haemangiomas [20]. The risk of needle induced bleeding during ultrasographic or CT guided biopsy in benign hpervascular tumors is reported to be low $(0.03 \%-0.04 \%)$ [17].

In this study none of our patient had liver biopsy preoperatively.

There is considerable controversy regarding the ideal treatment of giant haemangiomas of the liver. Some authors, on the basis of long-term follow up of such lesion, propse that most of these lesions do not require any treatment [21,22]. Other has treated these lesions surgerically, citing symptoms of the patient, increase in size and an occasional case of rupture, or diagnostic uncertainty [23].

Several therapeutic modalities are available for the treatment of haemangioma such as: steroids, interferon alfa-2 a, embolization, radiotherapy and surgery or liver transplantation. Resection of a hepatic haemangioma was first reported by Hermann Pfannestiel in 1898 and remains the only consistently effective method of treatment [24].

Irradiation therapy has been reported to provide partial reduction in size and relief of symptoms, but has risks, including radiation hepatitis, veno-occlusive disease, and hepatoma [4]. Because long-term effects of radiation therapy on the liver and adjacent structures are well documented, recommended radiation therapy only in patients who are unfit for or refuse surgery. Steroids have been used with some success in infants but their effectiveness in adults is not known [16]. Hepatic artery embolization has been used rarely for poor operative candidates, with some success in reducing the size of the lesion [25].

Arterial embolization should be considered for symptomatic patients in which resection is contraindication or before operation inpatients with a ruptured haemangioma, or even in elective cases, to reduce bleeding during resection [15].

The surgical options available for the treatment of giant haemangioma are hepatic artery ligation and resection either by enucleation or by formal liver resection, ligation of the hepatic artery can replace resectional treatment if the resection is considered too difficult or too risky [26].

Although most patients with haemangiomas of the liver may be safely followed up, some patients need surgery for symptoms (pain, etc.), large or increasing size, uncertain diagnosis and complication (consumptive coagulapathy, rupture etc.).

Yoon and associates [27] reported that the commonest indication for surgery was the presences of symptoms (60\%) other were an uncertain diagnosis (29\%) and large or increasing size (11\%). In another series from the US, [3] the indication of surgery were pain or increase in size (68\%), uncertain diagnosis (25\%) and rupture (7\%). In present series, the indications were pain (44\%), increasing in size (23.5\%) and uncertain diagnosis (20.5\%) and rupture (12\%).

Absolute surgical indications for hepatic haemangioma are spontaneous or traumatic rupture with haemoperitoneum, intratumoral bleeding, and consumptive coagulapathy (Kasabach-Merritt syndrome) persistent abdominal pain, obstructive jaundice, portal hypertension, superficial location of tumors larger than five $\mathrm{cm}$ with a risk of trauma, and an uncertain diagnosis are relative indication $[28,29]$.

Rupture of haemangioma with haemoperitoneum, is the most dreaded complication and often fatal if not promptly managed [30]. The first case of spontaneous rupture of a hepatic haemangioma was described by Van Haefen in 1898 in an autopsy case [31] in 1961, Swell and Weiss [32] reviewed 12 cases of spontaneous rupture of haemangioma from literature and reported the mortality rate to be as high as $75 \%$.

In the present study, 4 cases of rupture haemangioma received emergency liver resection to control the bleeding with no mortality.

In the current study, formal liver resection was chosen in 31 out of 34 patients, enucleatition in 2 patients and living related liver transplantation was selected for one patient, 1.5 years old, the size of multiple irresectable haemangiomas in both lobes was the indication for liver transplantation in this patient. No mortality, no recurrence was observed and the morbidity was comparable to that described by Imamura and associates [33].

An interesting finding in the present series was the absence of growth or complication from the haenmangiomas left in-situ in the liver remnants after resection, during an 18 months follow-up period. This underline the possibility of a tailored treatment for patients with symptomatic multiple haemangiomas in the liver.

\section{Conclusion}

Hepatic resection is a useful and effective treatment for giant cavernous haemangioma of the liver. More careful 
management to reduce intraopeative hemorrhage is recommended to increase the safety of surgery; in selected patients living related liver transplantation may be the only therapeutic option.

\section{REFERENCES}

[1] M. A. M. Brouwers, K. P. De Jong, E. B. Haagstmast, J. Klampmakert, M. A. Bijleveidt, J. H. Zwaveling and M. J. H. Sloot, "Surgical Treatment of Giant Haemangioma of the Liver," British Journal of Surgery, Vol. 84, No. 3, 1997, pp. 314-316.

[2] F. C. Nicholis, J. A. Van Heerden and L. H. Weiland, "Benign Liver Tumors," The Surgical Clinics of North America, Vol. 69, No. 2, 1989, pp. 297-314.

[3] R. Gedaly, J. J. Pomposelli, E. A. Pomfret, W. D. Lewis and R. L. Jenkins, "Cavernous Haemangioma of the Liver," Archives of Surgery, Vol. 134, 1999, pp. 407-411.

[4] O. Nishida, N. Satoh, A. S. Alam and J. Chino, "The Effect of Hepatic Artery Ligation for Irresectable Cavernous Haemangioma of the Liver," The American Surgeon, Vol. 54, No. 8, 1988, pp. 483-486.

[5] K. Tepetes, R. Selby, M. Webb, J. R. Madariaga, S. Iwatsuki and T. E. Starzl, "Orthotopic Liver Transplantation for Benign Hepatic Neoplasm,” Archives of Surgery, Vol. 130, No. 2, 1995, pp. 153-156.

[6] K. G. Ishak and L. Rabin, "Benign Tumors of the Liver," Medical Clinics of North America, Vol. 59, 1975, pp. 995-1013.

[7] Y. Cui, L. Y. Zhou, M. K. Dong, et al., "Ultrasonography Guided Percutaneous Radiofrequency Ablation for Hepatic Cavernous Haemangima," World Journal of Gastroenterlogy, Vol. 9, No. 9, 2003, pp. 2132-2134.

[8] T. Motohara, R. C. Semelka and L. Ragase, "MR Imaging of Benign Hepatic Tumors,” Magnetic Resonance Imaging Clinics of North America, Vol. 10, No. 1, 2002, pp. 1-14. doi:10.1016/S1064-9689(03)00046-1

[9] G. S. Deutsch, K. A. Yen, W. B. Bales, et al., "Embolization for Management of Hepatic Haemangiomas," The American Surgeon, Vol. 67, No. 2, 2001, pp. 159-164.

[10] S. N. Hochwald and L. H. Blungart, "Giant Haemangiomas with Kasabach-Merritt Syndrome: Is the Appropriate Treatment Enculation or Liver Transplantation?” HPB Surgery, Vol. 11, No. 6, 2000, pp. 413-419. doi:10.1155/2000/25954

[11] Y. Kumashiro, M. Kasahara, K. Nomoto, M. Kawai, K. Sasaki, T. Kiuchi and K. Tanaka, "Living Donor Liver Transplantation for Giant Hepatic Haemangioma with Kasabach-Merritt Syndrome with a Posterior Segment Graft,” Liver Transplantation, Vol. 8, No. 8, 2002, pp. 721-724. doi:10.1053/jlts.2002.33689

[12] P. J. Mego and P. R. Ros, "Benign Lesions of the Liver," Radiologic Clinics of North America, Vol. 36, No. 2, 1998, pp. 319-331.

[13] A. Cappellani, A. Zanghi, M. Divita, G. Zanghi, G. Tomarchio and G. Petrill, "Spontaneous Rupture of a Giant Haemangioma of the Liver," Annali Italiani Di Chirurgia, Vol. 71, No. 3, 2000, pp. 379-383.
[14] N. Coriglian, P. Mercantini, P. M. Amodio, G. Balducci, S. Caterino, G. Ramacciato, et al., "Hemoperitoneum from a Spontaneous Rupture of a Giant Heamangioma of Liver: Report of a Case,” Surgery Today, Vol. 33, No. 6, 2003, pp. 459-463. doi:10.1007/s10595-002-2514-Z

[15] S. Warmann, H. Bertram, R. Kardorff, M. Sasse, F. G. Hausdor and J. Fuchs, "Interventional Treatment of Infantile Hepatic Haemangioendothelioma,” Journal of Pediatric Surgery, Vol. 38, No. 8, 2003, pp. 1177-1181. doi:10.1016/S0022-3468(03)00325-7

[16] Q. L. Zeng, Y. H. Li, Y. Chen, Y. Ouyang, X. He and H. Zhang, "Gigantic Cavernous Haemangioma of the Liver Treated by Intra-Arterial Embolization with Pingyangmycin-Lipiodol Emulsion: A Multicentric Study,” Cardiovascular and Interventional Radiology, Vol. 27, No. 5, 2004, pp. 481-485.

[17] I. Ozden, A. Emre, A. Alper, M. Tunci, K. Acarli, O. Bilge, Y. Tekanty and O. Ariogul, "Long-Term Results of Surgery after Haemangiomas,” Archives of Surgery, Vol. 135, No. 8, 2000, pp. 978-981. doi:10.1001/archsurg.135.8.978

[18] E. J. Yun, B. I. Choi, J. K. Han, et al., "Hepatic Haemangioma: Contrast-Enhanced Pattern during the Arterial and Portal Venous Phases of Special CT,” Abdominal Imaging, Vol. 24, No. 3, 1999, pp. 262-266.

[19] T. Motohara, R. C. Semelka and L. Nagase, "MR Imaging of Benign Hepatic Tumors," Magnetic Resonance Imaging Clinics of North America, Vol. 10, No. 1, 2002, pp. 1-14.

[20] B. A. Birnabaum, J. C. Weinreb and A. J. Megibow, “Definitive Diagnosis of Hepatic Haemangiomas: MR Imaging versus Tc-99m-Labelled RBC,” Radiology, Vol. 126, 1990, pp. 95-101.

[21] T. Kerkivatan, W. W. Vrijland, P. T. Den Hoed, R. A. De Man, S. M. Hussain, H. W. Tilanus, et al., "Size of Lesion in Not a Criterion for Resection during Management of Giant Liver Haemangioma,” British Journal of Surgery, Vol. 89, No. 10, 2002, pp. 1240-1244. doi:10.1046/j.1365-2168.2002.02219.x

[22] T. Terkivatan, J. H. Dewilt, R. A. Deman, R. R. Van Rijn, P. E. Zondervan, H. W. Tilanus, et al., "Indication and Long-Term Outcome of Treatment for Benign Hepatic Tumors: A Critical Appraisal,” Archives of Surgery, Vol. 136, 2001, pp. 1033-1038. doi:10.1001/archsurg.136.9.1033

[23] H. P. Tsai, L. B. Jerg, W. C. Lee and M. F. Chen, “Clinical Experience of Hepatic Haemangioma Undergoing Hepatic Resection,” Digestive Diseases and Sciences, Vol. 48, No. 5, 2003, pp. 916-920.

[24] S. I. Schwatz and W. C. Husser, "Cavernous Haemangioma of the Liver; A Single Institution Report of 16 Resections,” Annals of Surgery, Vol. 205, 1987, pp. 456-465. doi:10.1097/00000658-198705000-00003

[25] P. Peveretos and D. Panoussopoul, “Giant Cavernous Hepatic Haemangioma: Treatment by Ligation of the Hepatic Artery,” Journal of Surgical Oncology, Vol. 31, No. 1, 1986, pp. 48-51. doi:10.1002/jso.2930310111

[26] I. Popescu, S. Ciuraa, V. Brasveanu, et al., "Liver Haemangioma Revisited: Currant Surgical Indication, Tech- 
nical Aspects, Results,” Hepatogastroenterology, Vol. 48, No. 39, 2001, pp. 770-776.

[27] S. S. Yoon, C. K. Charny, Y. Fong, W. R. Jamagin, L. H. Schwartz, L. T. Blumgart, et al., "Diagnosis, Manamgement, and Outcome of 115 Patients with Hepatic Haemangioma,” Journal of the American College of Surgeons, Vol. 197, No. 3, 2003, pp. 392-402. doi:10.1016/S1072-7515(03)00420-4

[28] A. Pietrabissa, P. Giulianotti, A. Campatelli, G. Di Candio, F. Farina, S. Signori, et al., "Management and Follow-Up of 78 Giant Haemangiomas of the Liver," British Journal of Surgery, Vol. 83, No. 7, 1996, pp. 915-918. doi:10.1002/bjs.1800830710

[29] A. E. Moreno, M. R. Del Pozo, M. C. Vicente and J. A. Abellan, "Indications for Surgery in the Treatment of Hepatic Haemangioma,” Hepatogastroenterology, Vol. 43,
No. 8, 1998, pp. 422-426.

[30] N. Corigliano, P. Mercantini, P. M. Amodio, G. Balducci, S. Caterino, G. Ramacciato, et al., "Haemoperitoneum from a Spontaneous Rupture of a Giant Haemangioma of the Liver: Report of a Case,” Surgery Today, Vol. 33, No. 6, 2003, pp. 459-463. doi:10.1007/s10595-002-2514-z

[31] V. Haefen, Inaugural Dissertation, Wurzbung, 1898.

[32] J. H. Sewell and K. Weiss, "Spontaneous Rupture of Haemangioma of the Liver. A Review of the Literature and Presentation of Illustrative Case," Archives of Surgery, Vol. 83, No. 7, 1961, pp. 29-33.

[33] H. Imamura, Y. Seyama, N. Kokudo, et al., "One Thousand Fifty-Six Hepatectomy without Mortality in 8 Years," Archives of Surgery, Vol. 138, No. 11, 2003, pp. 1198-1206. doi:10.1001/archsurg.138.11.1198 